\title{
Zur Frage der funktionellen Milzdiagnostik, nach Erfahrungen am entmilzten Menschen.
}

\author{
Von \\ Prof. Dr. Kreuter.
}

(Aus der Chirurgischen Klinik Erlangen [Direktor: Prof. Graser].)

(Eingegangen am 17. Dezember 1913.)

Der Begriff der "funktionellen Diagnostik der Milz" ist erst vor kurzem durch Frey in die experimentelle und klinische Medizin eingeführt worden. Er wurzelt in den Vorstellungen über den Einflu ß des vegetativen Nervensystems auf das Blutbild. Zur Diskussion steht zunächst die Frage, ob autonomtrope und sympathicotrope Gifte imstande sind, zu einer Verschiebung der morphologischen Blutelemente zu führen. Die Beantwortung dieser These durch die Autoren ist sehr verschieden. Man findet Zweifel an der Richtigkeit dieser Fragestellung überhaupt und entgegengesetzte Meinungen bei der Erörterung der Einzelheiten.

I.

Bertelli, Falta und Sch weeger glaubten trotz der großen Mannigfaltigkeit ihres experimentellen Tatsachenmaterials folgendes sagen $\mathrm{zu}$ können: Adrenalin führt von vornherein zu einem Überwiegen der Neutrophilen im peripheren Blut und später zu einer beträchtlichen Neutrophilie; Pilocarpin und andere autonomotrope Substanzen führen in einer ersten Phase - gleichgültig, ob Leukopenie oder Hyperleukocytose auftritt - zu einem Überwiegen der Lymphocyten und der großen Mononucleären. Später tritt ein völliger Umschlag ein mit Prävalenz der Neutrophilen. Diese Autoren kommen zur Aufstellung eines ,sympathicotonischen" Bluttypus im Sinne einer neutrophilen aneosinophilen Hyperleukocytose und eines ,,antonomotonischen “ Typus mit konstanter Mononucleose und sehr häufiger Hypereosinophilie; diese beiden Typen von Blutbildern finden sie in den verschiedensten pathologischen Zuständen immer wiederkehrend, und nur die Leukämien sollen von dieser Regel abweichen. Auch in den Versuchen von Schwenker und Schlecht macht Adrenalin echte Leukocytose mit Vermehrung der Neutrophilen; sie sahen aber auch Neutrophilie nach Pilocarpin ohne Lymphocytose. Skorczewski und Wasser- 
berg hinwieder beobachteten beim Menschen auf Adrenalin Zunahme der Neutrophilen ohne Leukocytose und auf Pilocarpin schon nach 20 Minuten Lymphocytose, die später in Neutrophilie übergeht.

Was die eosinophilen Zellen anlangt, so haben sich Neußer, Eppinger und Heß, Bertelli, Falta und Schweeger dafür ausgesprochen, daß Pilocarpininjektionen bei Kaninchen, Hunden und Menschen zu einer experimentellen Eosinophilie führen. Schwenker und Schlecht dagegen sahen beim Hund und Meerschweinchen eher eine Abnahme, bzw. ein Verschwinden der Eosinophilen; auch Stä ubli, Skorczews ki und Wasserberg erhielten durch Pilocarpin beim Menschen eine Hypereosinophilie. Die beiden letztgenannten Autoren hatten außerdem eine direkte chemische Reizwirkung der betreffenden Substanzen auf die blutbildenden Organe für viel wahrscheinlicher als den komplizierten Weg des negativen Nervensystems.

Anders liegen die Verhältnisse beim Adrenalin. Hier bestätigen Schwenker und Schlecht in Úbereinstimmung mit Bertelli, Falta und Schweeger, Skorczewski und Wasserberg nach Versuchen am Hund und am Meerschweinchen, daß die Eosinophilen zur Abnahme, ja sogar bis zum Verschwinden gebracht werden können und sich in der Leber anhäufen. Schwenker und Schlecht, ebenso Skorczewski und Wasserberg können jedoch darin keine spezifische negative Chemotaxis des Adrenalins auf die Eosinophilen erblicken, sondern sehen in dieser Erscheinung, ebenso wie Stä ubli, eine Reaktion des Blutes, die bei jeder Injektion körperfremder Substanzen entsteht.

Wenn bei gleicher Betrachtungsweise einer experimentellen Frage sich so erhebliche Differenzen in den Resultaten herausstellen, muß jeder Versuch, die Verhältnisse klarzustellen, dankenswert begrüßt werden. Dies gilt besonders auch für die Experimente von Fre y über den Einfluß des vegrtativen Nervensystems auf das Blutbild, zu welchen ihn klinische Gesichtspunkte veranlaßten, obwohl er die vorliegenden Arbeiten ,,nicht gerade ermunternd“ fand. Seine Versuche wurden an Kaninchen und Meerschweinchen ausgeführt. Adrenalin führte unmittelbar zu ener beträchtlichen Lymphocytose mit Rückgang der Neutrophilen; erst später, nach 45 Minuten, fallen die Lymphocyten wieder $a b$ und steigen die Neutrophilen an. Die abweichenden und nach Freys Ansicht irrtümlichen Angaben von Skorczewski und Wasserberg, von Bertelli, Falta und Schweeger sollen darauf beruhen, daß die Nachuntersuchungen erst ein'ge Stunden nach den Injektionen gemacht wurden. Auch bei Kaninchenversuchen mit dem ebenfalls sympathicotropen Diuretin sah Frey ganz regelmäßig erst eine L y mphoc y tose entstehen und später einen starken Abfall der Lymphocyten mit relativer polymorphkerniger Leukocytose ein- 
treten. Pilocarpin hatte bei Kaninchen in kleinen Dosen (1 $\mathrm{mg}$ ) zunächst keinen Effekt und führt nach 10 Minuten zu einer geringen Neutrophilie; größere Mengen $(5 \mathrm{mg}$ ) führen auch bei subcutaner Einverleibung sehr rasch zu einer Verdopplung der Lymphocytenwerte. Atropin erwies sich als nahezu wirkungslos.

Faßt man die Resultate der genannten. Autoren tabellarisch zusammen, so ergibt sich folgende Aufstellung:

Tabelle $\mathbf{I}$.

\begin{tabular}{|c|c|c|}
\hline Autoren & $\begin{array}{c}\text { Sympathicotrope Substanzen } \\
\text { machen }\end{array}$ & $\begin{array}{l}\text { A u t on o motrope substanzen } \\
\text { machen }\end{array}$ \\
\hline $\begin{array}{l}\text { Bertelli, Falta } \\
\text { und Schweeger }\end{array}$ & $\begin{array}{l}\text { Beträchtliche Neutrophilie. } \\
\text { Hyp-bzw. An-Eosinophilie. }\end{array}$ & $\begin{array}{l}\text { 1. Phase: überwiegen der Ly m- } \\
\text { phocy ten und groBenMo- } \\
\text { nonucleären. } \\
\text { 2. Phase: Umschlag in Neu- } \\
\text { trophilie. Hy per-Eosi- } \\
\text { nophilie. }\end{array}$ \\
\hline $\begin{array}{l}\text { Schwenker und } \\
\text { Schlecht }\end{array}$ & $\begin{array}{l}\text { Echte Leukocytose m. Ver- } \\
\text { mehrung der Neutrophilen. } \\
\text { Hyp- bzw. An - Eosinophilie. }\end{array}$ & $\begin{array}{c}\text { Neutrophilie ohne Lympho- } \\
\text { cytose. } \\
\text { Hyp- Eosinophilie. }\end{array}$ \\
\hline $\begin{array}{c}\text { Skorczewski } \\
\text { u. Wasserberg }\end{array}$ & $\begin{array}{l}\text { Zunahme der Neutrophilen } \\
\text { ohne Leukocytose. } \\
\text { Hyp-bzw. An-Eosinophilie. }\end{array}$ & $\begin{array}{c}\text { Ey mpocytose, spater Neu- } \\
\text { trophilie. } \\
\text { Hyp-Eosinophilie. }\end{array}$ \\
\hline Frey & $\begin{array}{c}\text { Unmittelbare beträchtliche } \\
\text { Lymphocytose, spater } \\
\text { Neutrophilie. } \\
\text { Hyp-Eosinophilie. }\end{array}$ & $\begin{array}{l}\text { Lymphocy tose. } \\
\text { Eosinophilie? }\end{array}$ \\
\hline
\end{tabular}

Frey vermehrt also die Wirkungsweise des Adrenalins insofern um ein bisher noch nicht beobachtetes Novum, als er unmittelbar nach der Injektion eine beträchtliche Lymphocytose entstehen sieht. Die eosinophilen Zellen sind nur in einer Tabelle berücksichtigt; aus zwei Versuchen geht hervor, daß sie unter Adrenalin abfallen.

Frey folgert wohl mit Recht, daß eine reaktive Lymphocytose nicht dụrch Ausbildung lymphocytärer Elemente zu erklären ist, sondern auf dem Wege einer mechanischen Mobilisierung aus dem lymphatischen Apparat stammen dürfte. Die Milz sei nun das hauptsächlich Lymphocyten bildende Organ. „Wenn die experimentelle Lymphocytose eine Milzreaktion ist, so war zu erwarten, daß sie nach Exstirpation des Organs ausbleibt." Bei milzexstirpierten Kaninchen fehlte nach Adrenalin- und Diuretininjektionen die Lymphocytose, das milzlose Meerschweinchen verhält sich wie ein normales Tier. Frey 
sieht also in der experimentell erzeugten Lymphocytose des Kaninchens die Folge einer Einwirkung der verwendeten Substanzen auf die glatte Muskulatur der Milz. „Damit ist der Antagonismus zwischen sympathischem und autonomem vegetativem System in bezug auf die Leukocyten des Blutes erledigt. Man braucht auch die Annahme einer Chemotaxis nicht. Der zu beobachtende Effekt ist in einfacher, eindeutiger Weise zu erklären durch eine mechanische Mobilisierung von lymphoiden Zellen in der Milz."

\section{II.}

Aus dem Ergebnis seiner Tierversuche glaubte sich Frey zu der Hoffnung berechtigt, in der Blutuntersuchung nach Adrenalin-, bzw. Pilocarpininjektionen eine brauchbare klinische Methode zu gewinnen, um Aufschluß über die Zusammensetzung und Funktionstüchtigkeit des Milzgewebes beim Menschen zu bekommen. ,Man konnte erwarten, daß je nach dem Gehalt, je nach der histologischen Beschaffenheit der Milz sich das Blutbild in entsprechender Weise verändern würde." In Gemeinschaft mit Lury untersuchte Frey zunächst eine größere Zahl von Menschen, bei denen keine Erkrankung der Milz anzunehmen war, um eigene Erfahrungen zunächst über ,"normale Verhältnisse zu sammeln.

Pilocarpin und Atropin zeigten keine nennenswerte Beeinflussung des Blutbildes. Es fehlt die Leukocytose, die relativen Zahlenschwankungeen entspechen nicht den absoluten. Die Eosinophilen zeigen Differenzen von $3 \%$, die innerhalb der Fehlergrenzen liegen. $\mathrm{Zu}$ diesem letzten Punkt ist vielleicht doch zu bemerken, daß regelmäBige, in derselben Richtung sich bewegende Schwankungen auch eine gewisse Beachtung verdienen und etwas Gesetzmäßiges haben können, wenn auch nur in subjektivem Sinne. Allein das subjektive Moment ist bei der Technik der Blutuntersuchung nie ganz zu eliminieren.

Adrenalin ergab eine ganz gesetzmäßige Verschiebung im Blutbild: in einer ersten Phase einen starken Anstieg der Lymphocytenwerte mit relativer Lymphocytose von durchschnittlich $12 \%$; in der zweiten Phase rascher Abfall der Lymphocyten; absolute und beträchtliche relative polymorphkernige Leukocytose. Die Befunde entsprechen denjenigen an Kaninchen und Meerschweinchen. Damit erwuchs für Frey und Lury ,die Berechtigung, analog dem Verhalten der milzexstirpierten Kaninchen auch beim milzkranken Menschen von der Norm abweichende Biesultate zu erwarten".

Die beiden Autoren untersuchten nun - unter Beschränkung a uf Adrenalininjektionen - eine große Reihe klinischer Fälle, bei denen Schädigungen der Milz anzunehmen oder sicher waren. Metastasierende Magencarcinome, Typhus, Anämien, Stauungsmilzen, Banti, 
Leukämie und Pseudoleukämie, schließlich auch nervöse Störungen wurden herangezogen. Die „Reaktion“ war bei Banti äußerst gering, im übrigen ,abhängig von der histologischen Beschaffenheit des Milzgewebes". Sie könne daher diagnostisch brauchbar sein bei Erkrankungen der Milz, über deren Bestand an funktionstüchtigem lymphoidem Gewebe sie Auskunft gibt. ,Sichere Anhaltspunkte für den Wert der Reaktion sind erst durch Untersuchung eines größeren Materials zu gewinnen."

\section{III.}

Wenn man die Arbeiten Freys studiert, kann man sich des Eindrucks nicht erwehren, daß seine Deduktionen etwas Sprunghaftes an sich haben. Ohne die aprioristische Annahme, daß die experimentelle Lymphocytose eine Milzreaktion sei, ist die ganze Fragestellung unverständlich. Daß die Befunde beim Kaninchen dafür, die beim Meerschweinchen nicht dafür, wenn nicht dagegen sprachen, mußte ernstlich zu bedenken geben, bevor man die Verhältnisse am Menschen angeht und zum Ausbau einer klinischen Methode sich anschickt. Man braucht gar nicht den chirurgischen Standpunkt und die vorliegenden Erfahrungen bei entmilzten Menschen zu betonen, sondern wird aus biologischen und physiologischen Erwägungen an den Versuchen Fre ys bemängeln müssen, daß die Milzexstirpation beim Tier in seinen Arbeiten als ein Faktum erscheint, das als solches durchaus nebensächlich behandelt wird und eine weiter nicht diskutierte Voraussetzung bildet. Man vermißt ernstlich die Besprechung der prinzipiell äußerst wichtigen Vorfrage, welchen Einfluß die Milzexstirpation für sich a uf das Blutbild hat. Man vermißt die Feststellung, ob, in welcher Zeit und auf welchem Wege sich das milzlose Tier in seiner Blutmorphologie auf den Verlust des Organs einstellt. Man findet ganz außer acht gelassen, daß die einzelnen Tierspezies und dies trifft besonders für das Kaninchen und Meerschweinchen zu mit dem Vorhandensein oder Fehlen von Blutlymphdrüsen vorzüghiche Ersatzorgane für die Milz besitzen oder nicht. Es ergeben sich somit eine Reihe von Gesichtspunkten, deren Erörterung ein logisches Bedürfnis ist, bevor es angängig erscheint, den Sprung zur ,funktionellen Milzdiagnostik" zu machen. Es ist auch etwas verwunderlich, daß Frey und Lury bei dem Gange der ganzen Versuchsmethodik von der "Untersuchung eines größeren Materials" sichere Anhaltspunkte über den Wert der Reaktion erwarten. Die näherliegende und heutzutage durchaus nicht unerfüllbare Forderung, die Frage am entmilzten Menschen nachzuprüfen, wird nicht ausgesprochen. Es bedarf keiner Worte, um festzulegen, daß dies die einzig sichere und einwandfreie Möglichkeit ist, dem an sich zweifellos interessanten Problem näherzutreten. Dabei muß selbstverständlich die unabweisbare 
Voraussetzung erfüllt sein, daß es sich um Individuen handelt, welche die normale Milz verloren haben. Es kommen also nur die Menschen in Betracht, bei denen wegen traumatischer Ruptur die histologisch gesunde Milz entfernt werden mußte.

Wer an Blutexperimente beim entmilzten Tier und Menschen herangeht, wird zuerst den Einfluß des Organverlustes a uf das Blutbild festzustellen haben. Ganz besonders die Erfahrungen beim Menschen lehren. eindringlich, $\operatorname{da} \beta$ hier noch vieles im Fluß ist und der definitiven Feststellung bedarf. Es ist hier nicht der Ort, um auf diese interessanten Punkte einzugehen. Ich verweise auf meine klinische Arbeit über das Blutbild nach der Milzexstirpation, die demnächst in Bruns' „Beiträgen zur klinischen Chirurgie“ erscheint; auch über Experimente an Affen werde ich später berichten. Hier möchte ich nur kurz auf den Fall eingehen, der mir eine willkommene Veranlassung gab, mich mit der Frage der funktionellen Milzdiagnostik zu beschäftigen. Die klinischen Daten sind folgende:

Ein 15 jähriger Lehrling stürzte $3 \mathrm{~m}$ hoch ab und fiel mit der linken Seite auf einen Balken. Er hatte heftige Schmerzen an der Stelle der Verletzung und wurde in ein städtisches Krankenhaus gebracht. Im Laufe der Nacht entwickelte sich Meteorismus und Erbrechen. Der zuständige Arzt veranlaßte die Einlieferung in die chirurgische Klinik Erlangen. Wir fanden so schwere Erscheinungen einer subcutanen Bauchkontusion, daß sofort die Laparotomie gemacht wurde (Prof. Graser). In der Bauchhöhle war schätzungsweise 1 Liter flüssiges Blut und lagen dicke Blutklumpen. Neben einem $\mathrm{Ri} \beta$ im Mesokolon stellte sich eine Zertrümmerung der Milz heraus. Sie wurde exstirpiert, die Bauchwunde bis auf eine kleine Drainagelücke geschlossen. Abgesehen von einem pneumonischen Infiltrat erfolgte primäre Wundheilung. Nach 18 Tagen konnte der sonst kräftig entwickelte junge Mensch entlassen werden. Die exstirpierte Milz war histologisch normal.

Es handelte sich somit um einen Fall, bei dem einem gesunden jungen Menschen die Milz wegen totaler Zerreißung herausgenommen werden mußte. Als er - nicht ganz 3 Wochen nach der Operation und nach ungestörter Wundheilung - zur Entlassung kam, hatte er folgendes Blutbild:

Erythrocyten: 1140 000; Leukocyten: 22500 (!). Polymorphkernige: . . . . . absolut 9450 ; relativ $42 \%$; Lymphocyten . . . . . . . . , 12375 ; , $55 \%$; Eosinophile . . . . . . . " " $112 ; \quad, \quad 0,5 \%$; Mononucleäre u. Übergangszellen . ", $\quad 562 ; \quad, \quad 2,5 \%$.

Ferner fand sich in 300 Feldern ein Erythroblast.

Dieses Blutbild kann als charakteristisch angesehen werden für eine un ko mplizierte Milzexstirpation. Der starke AbfallderEr y throcyten entspricht dem Blutverlust. Dei beträchtliche Hyperleukocystose ist eine spezifische Folgeerscheinung der Milzexstir- 
pation (Michelssohn). Sie kann jahrelang anhalten (NoetzelApolant, Riegner). Bemerkenswert sind an unserem Fall dieniedrigenZahlen derNeu tro philenund diestarkeL y m phoc ytose, letztere ein Ausdruck für die enorme Mehrleistung der vikariierend eintretenden Lymphdrüsen. Eine damit einhergehende Schwellung der peripheren Lymphknoten (Krabbel, Trendelenburg u. a.) oder Schmerzen in den Diaphysen der Röhrenknochen infolge der Knochenmarksreizung (Rieg ner) konnten wir nicht feststellen. Hervorzuheben ist auch, daß die Eosinophilen nur 0,5\% betragen, da sie sonst, d.h. später nach Milzverlust regelmäßig vermehrt auftreten (Ehrlich).

Sechs Wochen nach der Milzexstirpation ließen wir den Kranken wieder kommen, um ihn ,funktionell' zu prüfen. Er erfreute sich besten Wohlseins und hatte sich glänzend erholt. Wir hielten uns genau an die Versuchsanordnung von Frey und Lury. Adrenalin wurde in der Menge von 0,001 , Pilocarpin 0,01, beides subcutan, verabreicht. Kurz vor den Injektionen, $1 / 2$ und' 1 Stunde danach wurden die Blutentnahmen gemacht und die einzelnen Zählungen mehrfach kontrolliert ${ }^{1}$ ). Das Individuum war stets nüchtern, um Verdauungsschwankungen im weißen Blutbild auszuschalten.

Überraschend warvorerst die Tatsache, daßsich 6 Wochen nach dem starken Blut-und vollkommenen Milzverlust das morphologische Blutbild nahezu vollständig wiederhergestellt hatte. Die Zählung ergab:

Erythrocyten: 5260 000; Leukocyten: 8700;

Polymorphkernige. . . . . . absolut 5655; relativ $65 \%$;

Lymphocyten . . . . . . . " 1740; " 20\%;

Eosinophile: . . . . . . . " , $452 ; \quad, \quad 5,2 \%$;

Mononucleäre und Ưbergangszellen . ", $853 ; \quad, \quad 9,6 \%$.

Der Hämoglobingehalt des Blutes betrug jedoch nur $57 \%$. Dieser Hämoglobinmangel ist vielleicht die einzige, bisher sichergestellte Ausfallserscheinung nach Milzverlust. Wenm man von der neuerdings behaupteten Bedeutung des Organs für die Tu morimmunität absieht, ist nur von Groß eine Störung in der Pepsinverdauung durch Wegfall eines Hormons angenommen worden. Dagegen haben Asher und seine Schüler, ferner Ba yer u. a. einwandfrei festgestellt, daß die Milz im Eisenhaushalt des Organismus eine fixe Rolle spielt. Mit ihrem Verlust setzt eine gesteigerte Eisenabscheidung ein; Eisen, das im Stoffwechsel frei wird, geht dem Organismus

1) Um diese Untersuchungen hat sich Frl. Dr. Ditt mar in hingebender Weisse bemüht. Sie hatte keinerlei Kenntnis von dem Zweck und Ziel ihrer Aufgabe, so daß ihre Resultate Anspruch auf weitgehendste Objektivität beanspruchen können. 
verloren und kann in den roten Blutzellen nicht zur Aufspeicherung kommen.

Die Bedingungen zur Nachprüfung der Frage der funktionellen Milzdiagnostik waren somit unerwartet günstig, da ein milzloser Mensch mit nahezu normalem Blutbild zur Verfügung stand. Es gab also keine Nebenfrage zu berücksichtigen und die „Milzreaktion“ konnte nur eindeu tig ausfallen. Darüber gibt die folgende Zusammenstellung Aufschluß:

Tabelle II.

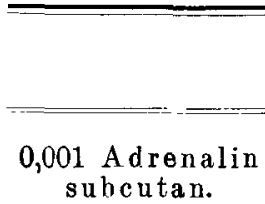

1. Nov. Vor der Injektion . . . $1 / 2$ Std. nach der Injektion .

1 Std. nach der Injektion .

2. Nov. Vor der Injektion. . .

$1 / 2$ Std. nach der Injektion . .

1 Std. nach der Injektion . 0,01 Pilocapin subcutan.

3. Nov. Vor der Injektion. . $1 / 2$ Std. nach der jektion. .

1 Std. nach der Injektion .

\begin{tabular}{|c|c|c|c|c|c|c|c|c|c|c|}
\hline \multirow[t]{2}{*}{$\begin{array}{c}\text { Leuko- } \\
\text { cyten }\end{array}$} & \multicolumn{2}{|c|}{ Polymorph. } & \multicolumn{2}{|c|}{$\begin{array}{l}\text { Lympho- } \\
\text { cyten }\end{array}$} & \multicolumn{2}{|c|}{\begin{tabular}{|c|} 
Eosino- \\
phile
\end{tabular}} & \multicolumn{2}{|c|}{$\begin{array}{l}\text { Mast- } \\
\text { zellen }\end{array}$} & \multicolumn{2}{|c|}{$\begin{array}{l}\text { Monucleär. u } \\
\text { tbergangsz. }\end{array}$} \\
\hline & abs. & $\%$ & abs. & $\%$ & abs. & $\%$ & abs. & | \% & abs. & $\%$ \\
\hline 8700 & 5655 & 65 & 1740 & 20 & 452 & 5,2 & - & - & 835 & 9,6 \\
\hline 9200 & 4600 & 50 & 2760 & 30 & 828 & 9,1 & 83 & 0,9 & 920 & 10,4 \\
\hline 10400 & 6032 & 58 & 2558 & 24,6 & 384 & $3, y$ & 62 & 0,6 & 1352 & 13,0 \\
\hline 9800 & 5292 & 54 & 2842 & 29 & 822 & 8,3 & - & - & 822 & 8,3 \\
\hline 11400 & 3192 & 28 & 6338 & 55,6 & 752 & 6,6 & - & - & 1037 & 9,1 \\
\hline 18000 & 11340 & 63 & 4500 & 25,0 & 900 & 5,0 & 126 & 0,7 & 1134 & 6,3 \\
\hline 9400 & 5734 & 61,7 & 2162 & 23,0 & 573 & 6,1 & 56 & 0,6 & 752 & 8,0 \\
\hline 8200 & 4182 & 51,0 & 2312 & 28,2 & 779 & 9,5 & -1 & - & 918 & 11,2 \\
\hline 9400 & 5245 & 55,8 & 2303 & $\mid 24,5$ & 893 & 9,5 & - & -1 & 996 & 10,6 \\
\hline
\end{tabular}

Aus dieser Tabelle läßt sich ablesen, daß Adrenalin eine sehr beträchtliche Leukocytose verursacht, die fast zur Verdopplung der Zahlen führt. In einer ersten Phase - $1 / 2$ Stunde nach der Injektion - entsteht eine a usges prochene Ly m phoc y tose mit Differenzen bis zu $26 \%$, welche in der zweiten Phase - 1 Stunde nach der Injektion - in Neutrophilie umschlägt. Die Zahlen der Eosinophilen gehen in unverkennbarer Weise zur ück. Daß die Ausschläge 
am zweiten Versuchstag noch weit mehr in die Augen springen als am ersten, hängt wohl mit einer kumulierenden Wirkung des Adrenalins zusammen.

Pilocarpin verändert das Blutbild sehr wenig und ohne Prägnanz. Am meisten tritt noch ein Ansteigen der Eosinophilen hervor.

Es bedarf keines besonderen Scharfsinns, um gegen diese Versuche und ihre Ergebnisse eine Reihe von Einwendungen zu machen. Schon ihre geringe Zahl hat ihre Bedenken. Auch der Umstand, daß sie an drei aufeinanderfolgenden Tagen angestellt wurden, entspricht nicht allen Anforderungen an die Exaktheit. Allein daran sind lediglich äußere Verhältnisse schuld. Unser Objekt ließ sich durch nichts länger halten. Die sachliche Beschränkung wurde somit aufgezwungen. Daß die Mitteilung trotzdem ihre Berechtigung hat, ergibt das wissenschaftliche Interesse an der Fragestellung und die selten günstige Gelegenheit, sie am Menschen unter beweiskräftigen Voraussetzungen zu erörtern, von selbst.

Trotz aller Einschränkungen, zu welchen eine gesunde Kritik gegenüber den vorliegenden Resultaten berechtigt ist, zeigen unsere Blutuntersuchungen doch bemerkenswerte Punkte. Sie decken sich nahezu vollständig mit den von Frey und Lury für den normalen, d. h. milzgesunden, Menschen gegebenen Bluttypen. Namentlich die von den genannten Autoren zuerst beschriebene primäre Lymphocytose nach Adrenalingaben ist sehr eklatant. Da unsere Befunde jedoch vom entmilzten Menschen stammen, müssen sie zu einer strikten Ablehnung der „funktionellen Milzdiagnostik" führen. Es ist natürlich sehr wünschenswert, auf diesemeinwandfreien Wege noch weitere Erfahrungen zu sammeln, deren Ergebnis jedoch kaum zweifelhaft sein kann.

Wenn nun ein entmilzter Mensch in relativ kurzer Zeit sein Blutbild wieder nahezu ganz regenerieren kann, so wird man dadurch von neuem auf die Organe aufmerksam, die vikariierend für die Milz eintreten können. In dieser Hinsicht sind unsere Kenntnisse für den Menschen noch sehr lückenhaft. Daß Nebenmilzen hypertrophieren und ersetzend eintreten können, ist das Naheliegendste. Die Frage der Milzregeneration aus zurückgebliebenen Organresten (Küttner) muß mancherlei prinzipiellen biologischen Bedenken begegnen. Inwieweit beim Menschen Blutlymphdrüsen (Faltin) in Betracht kommen, und welche Bedeutung die ,Splenoide" v. Stubenrauchs haben, steht noch dahin. Die einzelnen Tierarten verhalten sich in bezug auf die Ersatzmöglichkeiten so verschieden, daß sie zur Beurteilung menschlicher Verhältnisse nur mit größter Vorsicht herangezogen werden dürfen. Diesen dürften uns Experimente am Affen noch am nächsten bringen. 
Es reagiert also der milzlose Mensch auf die Injektion autonomotroper und sympathicotroper Substanzen in der gleichen Weise wie das milzgesunde Individuum; eine ,funktionelle Diagnostik der Milz" im Sinne von Frey und Lury ist daher a uf diesem Wegenicht möglich. Die Verschiebungen im peripheren Blutbild werden durch den Ausfall der Milz nicht beeinflußt.

\section{Literaturverzeichnis.}

Bertelli, Falta u. Schweeger, Über die Wechselwirkung von Drüsen mit innerer Sekretion. Zeitschr. f. klin. Medizin y1. 1910.

Ba y er, Untersuchungen über den Eisenstoffwechsel. Mitt. a. d. Grenzgeb. d. Med. u. Chir. 21. 1910.

Faltin, R., Milzartige Bildungen im Peritoneum usw. Deutsche Zeitschr. f. Chir. 110. 1911.

Frey, W., Der Einfluß des vegetativen Nervensystems auf das Blutbild. Zeitschr. f. d. ges. experim. Med. 2. 1913.

- u. S. Lury, Adrenalin zur funktionellen Diagnostik der Milz? Ebda.

Michelssohn, F., Die Ergebnisse der modernen Milzchirurgie. Ergebn. d. Chir. u. Orthop. 6. 1913.

Schwenker, G., u. H. Schlecht, Über den Einfluß sympathico- und autonomotroper Substanzen auf die eosinophilen Zellen. Zeitschr. f. klin. Medizin 76. 1912.

Skorczewski u. Wasserberg, Besteht ein Zusammenhang zwischen der Reizung des $N$. vagus und des $N$. sympathicus einerseits und der unter der Wirkung spezifischer Gifte veränderten Zusammensetzung des Blutes andererseits? Zeitschr. f. experim. Pathol. u. Ther. 10. 1912.

Stä ubli, Die klinische Bedeutung der Eosinophilie. Ergebn. d. inn. Med. u. Kinderheilk. 6. 1910.

v. St u be n ra u ch, Milzregeneration und Milzersatz. Verhandl.d. Deutsch. Gesellsch. f. Chir. Jg. 41. 1912. 